\title{
Paradoxe identitaire et interdisciplinarité : un regard sur les identités disciplinaires des chercheurs
}

\author{
Ayuko Sedooka ${ }^{1}$, Gabriela Steffen ${ }^{2}$, Theres Paulsen ${ }^{3}$, Frédéric Darbellay ${ }^{4}$ \\ 1 Sciences de l'éducation, Université de Genève, Centre interfacultaire en droits de l'enfant, 1950 Sion, Suisse \\ 2 Linguistique, Université de Genève, Centre interfacultaire en droits de l'enfant, 1950 Sion, Suisse \\ 3 Sciences de l'environnement, Académies suisses des sciences, td-net, Network for Transdisciplinary Research, 3007 Berne, Suisse \\ 4 Interdisciplinarité, Université de Genève, Centre interfacultaire en droits de l'enfant, Cellule Inter- et Transdisciplinarité, \\ 1950 Sion, Suisse
}

\begin{abstract}
NSS, qui se veut avec détermination et constance une revue scientifique interdisciplinaire, a souvent publié des articles, « Regards » ou «Vie de la recherche » sur l'interdisciplinarité. Si de nombreux textes, factuels ou normatifs, sont à base de réflexions individuelles ou collectives, si d'autres sont des comptes rendus critiques de colloques ou de livres, les articles de recherche sur la pratique sociale de l'interdisciplinarité, à base d'enquêtes de terrain, de questionnaires, d'entretiens libres ou semi-directifs, ou de débats en «focus groups » sont plus rares et précieux. Ce travail, issu d'une recherche sur contrat avec une population assez considérable et diverse, et s'appuyant sur les cadres conceptuels sociologiques de la construction des identités, présente l'intérêt d'aborder la question du point de vue de l'affirmation et de la perception par les chercheurs des identités disciplinaires et interdisciplinaires, dans les contextes de relations avec les partenaires de recherche et avec les communautés scientifiques d'appartenance. L'étude est attentive aux paradoxes, aux dynamiques et aux évolutions que manifestent ces identités.
\end{abstract}

La Rédaction

Mots-clés :

recherche ;

dispositifs

institutionnels ;

identité disciplinaire ; interdisciplinarité ; trajectoire académique

\begin{abstract}
Résumé - Cette contribution vise à s'interroger sur les identités disciplinaires de chercheurs qui pratiquent la recherche interdisciplinaire. Nous tentons de mettre en évidence la nature paradoxale du travail interdisciplinaire au travers des regards que portent des chercheurs sur leur propre identité dans le contexte académique. Cette nature paradoxale correspond à une tension entre, d'une part, des identités disciplinaires relativement stables et institutionnellement reconnues et, d'autre part, des identités interdisciplinaires hybrides qui doivent encore obtenir une forme de reconnaissance. Nous proposons une typologie de différents profils types qui se déroule sur un continuum allant de la disciplinarité à l'indisciplinarité : l'identité disciplinaire ; les identités interdisciplinaires, déclinées en identité thématique, identité hybride, «interdisciplinary native » et «interdisciplinary migrant »; et enfin l'identité indisciplinée. Notre questionnement s'appuie sur les résultats d'une recherche menée sur les pratiques de recherche interdisciplinaire.
\end{abstract}

\begin{abstract}
Identity paradox and interdisciplinarity: an analysis of the disciplinary identities of researchers. This contribution proposes to investigate the disciplinary identities of researchers involved in interdisciplinary research in order to highlight its paradoxical nature which is revealed in their discourse about the way they perceive themselves and their own identity in the academic context. This paradoxical nature results in a tension between relatively stable and institutionally recognized disciplinary identities on the one hand and interdisciplinary hybrid identities that are still in need of recognition on the other. We address the complex relationship between the apparently stabilized disciplinary identity of researchers and their interdisciplinary research practices that lead them to redefine their identities in contact with other disciplines in a dynamic of multiple perceptions and
\end{abstract}

Auteur correspondant : A. Sedooka, ayuko.sedooka@unige.ch 


\section{Keywords:} research; institutional framework; disciplinary identity; interdisciplinarity; academic path representations. How do researchers demonstrate and represent their disciplinary -in some cases interdisciplinary or even undisciplinary- identity? What are their relations with their institutional belonging and respective disciplines? What are their academic and career paths that guide their practices? By exploring these issues we propose a typology of different type-profiles of researchers who practice interdisciplinary research. The typology runs on a continuum from disciplinarity to undisciplinarity: disciplinary identity; interdisciplinary identities expressed as thematic identity, hybrid identity, "interdisciplinary native" and "migrant interdisciplinary"; and finally undisciplined identity. These reflections and typology are based on the results of a study on interdisciplinary research practices.

\section{Pratiques de recherche interdisciplinaire et paroles de chercheurs}

Les pratiques de recherche interdisciplinaire mettent en contact non seulement des savoirs issus de diverses disciplines mais aussi, et surtout, des chercheurs venant d'horizons disciplinaires, culturels et linguistiques différents (Lemay et Darbellay, 2014 ;Steffen et al., 2015). La notion de discipline est doublement intéressante pour une analyse des pratiques de recherche interdisciplinaire. Elle désigne à la fois une « catégorie organisationnelle au sein de la connaissance scientifique » (Morin, 1990, p. 5) et les « règles de conduite imposées qui cadrent (disciplinent) les pratiques d'apprentissage auxquelles tout élève doit se soumettre en faisant acte d'obéissance et d'obédience » (Origgi et Darbellay, 2010, p. 7). Les nombreuses études sur l'interdisciplinarité, dont il ne s'agit pas ici de faire la revue, se sont souvent centrées sur ce qu'apportent les disciplines, mais plus rarement sur les personnes qui les mettent en œuvre en tant que chercheurs-acteurs académiques, en leur donnant une parole réflexive ${ }^{1}$. Des études comme celle de Brun et ses collègues (Brun et al., 2007) sur les postures des chercheurs mettant en place l'interdisciplinarité dans le domaine de l'environnement montrent justement tout l'intérêt d'une autoanalyse réflexive basée sur leurs propres expériences de recherche interdisciplinaire. Dans la même veine, l'ouvrage de Mougenot (2011) présente et analyse des visions ancrées dans le vécu de chercheurs actifs dans le domaine interdisciplinaire de l'environnement et de la biodiversité. Elle souligne que « l'interdisciplinarité dont ils parlent n'est pas seulement celle des autres ", mais bien la leur qui se donne à entendre et à lire par la « force du récit ». Le travail collectif édité par Mathieu et Schmid (2014) va dans ce même sens d'une " forme directe du discours », en donnant la parole à douze scientifiques issus de six disciplines (anthropologie, biologie, géographie, linguistique, philosophie,

1 Il convient à ce titre de mentionner la rubrique « Regards » de la revue Natures Sciences Sociétés (NSS) qui propose régulièrement des contributions sur des retours d'expériences et des témoignages de chercheurs engagés dans le travail interdisciplinaire. physique) et qui témoignent de leurs démarches de recherche et du lien qu'ils tissent entre interdisciplinarité et modélisation. Tout chercheur ${ }^{2}$ est, en effet, à la fois acteur et observateur de ses propres pratiques interdisciplinaires : il porte un regard réflexif sur son travail individuel et collectif, sur son identité et celle de sa communauté scientifique d'appartenance. Ce regard peut être compris comme une forme $\mathrm{d}^{\prime}$ « anthropologie chez soi » qui explore l'appartenance culturelle, professionnelle, linguistique ou sociale du chercheur dans son rapport à ses objets et terrains d'étude (Gullestad et al., 2009 ; Ouattara, 2004). Dans cette perspective, le terrain d'enquête des pratiques de recherche interdisciplinaire est à la fois un objet d'étude pour notre équipe de recherche dont nous analysons et exposons ici certains résultats, tout en offrantl'occasion d'une autoréflexion sur nos propres pratiques et vécus scientifiques qui devrait nourrir le débat sur les enjeux de l'interdisciplinarité.

La présente contribution vise à interroger l'approche réflexive des chercheurs sur leurs appartenances et leurs identités disciplinaires lorsqu'ils pratiquent la recherche interdisciplinaire. Elle se base sur les résultats d'une étude de cas multiples menée dans le cadre du projet de recherche "Analyzing Interdisciplinary Research : From Theory to Practice. Case Studies in the Swiss University Context $»^{3}$. Ce projet analyse des pratiques de recherche interdisciplinaire, en s'intéressant en particulier à la manière dont l'interdisciplinarité est mise en œuvre par les chercheurs dans divers contextes académiques. Dix structures/institutions (centres, laboratoires, etc.), dont cinq suisses romandes et cinq suisses allemandes, ont été choisies comme cas d'étude ${ }^{4}$. Sans

\footnotetext{
2 Dans le présent document, l'utilisation du genre masculin a été adoptée afin de faciliter la lecture et n'a aucune intention discriminatoire.

3 Le projet a été financé par le Fonds national suisse de la recherche scientifique (Requête n CR11I1_143816), pendant deux ans (2014-2015). Équipe de recherche: F. Darbellay (requérant principal), P. Perrig-Chiello et A.-C. Berthoud (co-requérantes), A. Sedooka, T. Paulsen et G. Steffen.

4 Les institutions d'appartenance et les personnes sont ici anonymisées.
} 
viser une quelconque représentativité statistique de la recherche interdisciplinaire, cette sélection a été réalisée sur la base de la visibilité en matière d'interdisciplinarité de ces institutions dans le paysage académique suisse, travaillant explicitement sur des thématiques qui se situent à la croisée de plusieurs disciplines (environnement et développement durable, études des migrations, santé, etc.).

Une attention particulière a été portée à la diversité des institutions. Ceci permet de montrer une certaine variation dans la manière de penser et de mettre en œuvre différentes formes de recherche dans des thématiques variées. Au total 66 chercheurs sélectionnés dans ces dix structures universitaires ont participé à l'enquête, en passant au filtre d'un dispositif méthodologique "mixte", qui combine des approches qualitatives et quantitatives avec en l'occurrence une dominance des méthodes qualitatives (Creswell et Plano Clark, 2007 ; Tashakkori et Teddlie, 2003). Le dispositif comprend un questionnaire (65 répondants), des entretiens semi-directifs (trente, soit trois entretiens par cas) et un focus group par cas (dix focus groups de quatre à sept chercheurs) ${ }^{5}$. La sélection des personnes interrogées a tenu compte des critères de diversité des statuts académiques, des appartenances disciplinaires, de genre et d'âge.

L'analyse des données recueillies a été organisée en cinq dimensions interdépendantes qui couvrent les principaux enjeux des pratiques de recherche interdisciplinaire : (I) le contexte institutionnel ; (II) la motivation, l'identité disciplinaire et la trajectoire des chercheurs ; (III) les réflexions épistémologiques, théoriques et méthodologiques sur l'interdisciplinarité ; (IV) les formes de collaboration entre les chercheurs ; (V) les publications et l'évaluation de la recherche interdisciplinaire ${ }^{6}$. Nous n'abordons pas ici la totalité de ces dimensions pour focaliser notre attention sur la question de l'identité des chercheurs qui se lit dans leurs différentes trajectoires académiques dans un contexte interdisciplinaire. Nous problématisons les rapports complexes entre les ancrages disciplinaires apparemment stabilisés des chercheurs et leurs pratiques de recherche interdisciplinaire au travers desquelles ils tendent à redéfinir leurs identités au contact des autres disciplines, dans un jeu de perceptions et de représentations multiples. Comment les chercheurs témoignent-ils de leur identité disciplinaire, voire dans certains cas proprement (inter)disciplinaire ou même indisciplinée, et se la représentent-ils? Comment se situent-ils dans leur contexte institutionnel

5 Le questionnaire, les entretiens et les focus groups ont été réalisés en français et en allemand. Les citations extraites du corpus germanophone ont été traduites en français par nos soins.

6 L'analyse de l'ensemble de ces dimensions sera présentée dans un ouvrage à paraître en 2016. d'appartenance, notamment par rapport à leur ancrage disciplinaire? Quels sont les parcours académiques et professionnels qui guident leurs pratiques? L'exploration de ces questions débouche sur une proposition de typologie de différents profils types identitaires des chercheurs qui pratiquent la recherche interdisciplinaire.

\section{Appartenances et identités disciplinaires}

Dans la vie professionnelle des chercheurs, l'identité disciplinaire est une forme d'inscription marquante et constitutive. Clark (1983) définit les disciplines académiques en tant que structures institutionnelles qui constituent les communautés de base dans lesquelles les chercheurs construisent leur propre identité professionnelle. Les institutions universitaires s'organisent en général en disciplines pour assurer l'élaboration et la transmission de connaissances spécialisées. La notion de discipline a alors une signification forte pour la plupart des chercheurs qui appartiennent à des «tribus académiques »(Becher et Trowler, 2001). En outre, ces mêmes institutions entendent promouvoir et encourager l'enseignement et la recherche interdisciplinaires. Les enseignants-chercheurs qui souhaitent s'inscrire dans cette tendance sont parfois pris dans une tension entre la volonté de s'ouvrir et de répondre aux attentes des discours institutionnels et la persistance de structures largement organisées en disciplines et sources de difficultés (Wainwright et al., 2014). Dans un tel contexte institutionnel, politiquement favorable à l'interdisciplinarité mais structurellement difficile pour sa mise en œuvre, la question de l'identité disciplinaire des chercheurs devrait faire l'objet d'un débat central, non seulement dans les communautés disciplinaires, mais aussi dans les communautés interdisciplinaires. Les chercheurs qui entendent s'ouvrir au dialogue, voire au dépassement des frontières entre les disciplines, doivent se confronter à la tendance inverse d'un « enfermement disciplinaire » dans lequel est souvent ignorée la richesse des interfaces entre les disciplines (Charbonneau, 2005). Ce fait est fréquemment doublé d'un « ethnocentrisme disciplinaire » caractérisé par une attitude de fidélité extrême envers une discipline d'appartenance (Campbell, 1969).

Dans notre recherche, la question de l'appartenance disciplinaire des chercheurs est abordée de deux manières par l'enquête : indirecte et directe. Dans une première phase de l'enquête, nous avons pu identifier les ancrages disciplinaires de l'ensemble des chercheurs. Cette détermination indirecte des identités disciplinaires a été réalisée sur la base des données récoltées dans la documentation officielle des institutions concernées, ainsi que sur la base des informations figurant sur les sites web. Tous les chercheurs ont également été consultés 


\section{Disciplines Nombre de personnes}

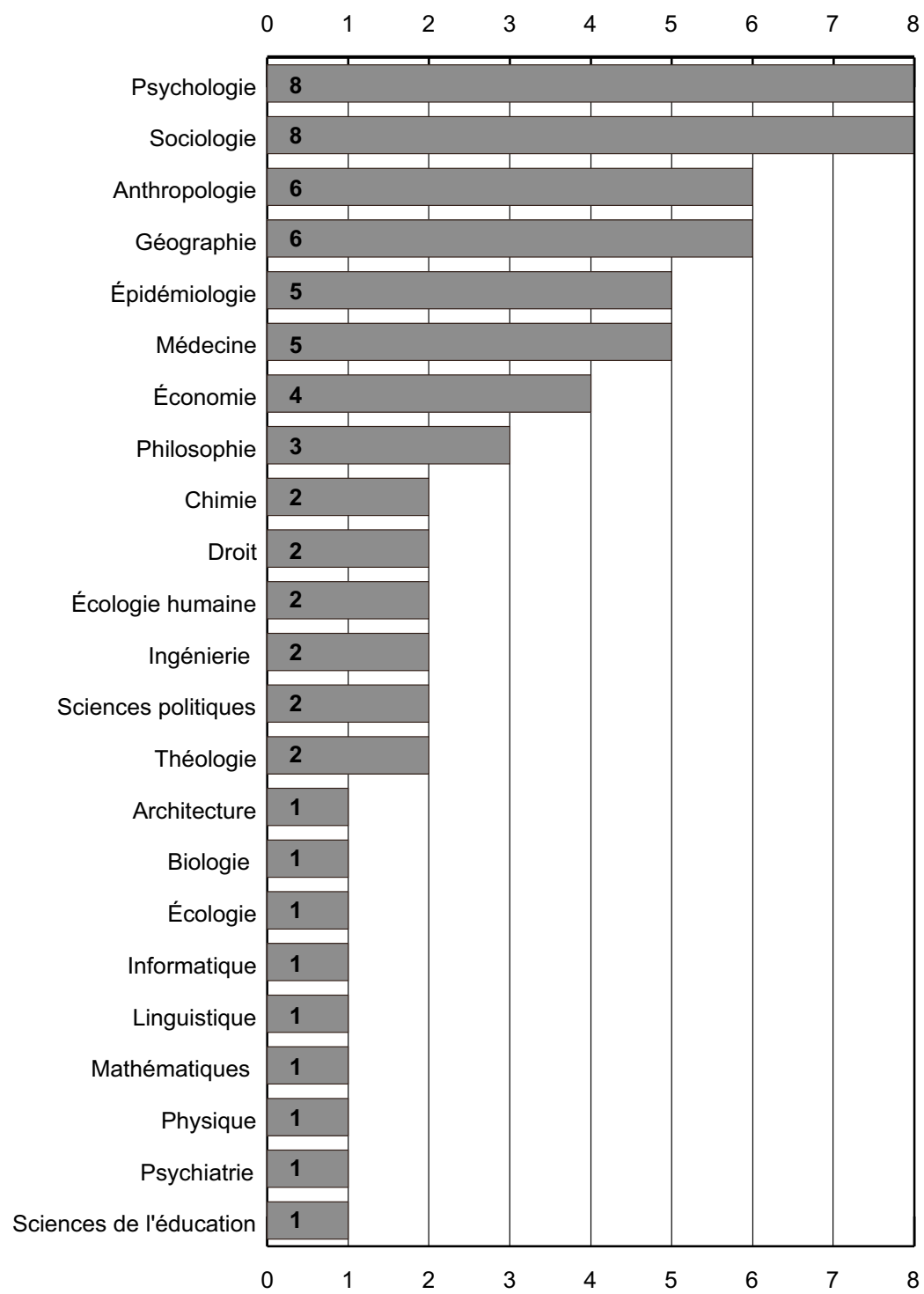

Fig. 1. Identités disciplinaires des participants-chercheurs $(\mathrm{N}=66)$.

directement à ce sujet et ils ont à l'unanimité confirmé leur identification disciplinaire de base.

Les ancrages disciplinaires des participants-chercheurs de notre enquête (Fig. 1) montrent une prégnance de quatre disciplines (la psychologie, la sociologie, l'anthropologie et la géographie), suivies de près par la médecine et l'épidémiologie. Les autres ancrages disciplinaires se déclinent en un large spectre qui va de l'économie au droit, en passant par la chimie, la biologie, les mathématiques ou encore les sciences de l'éducation. Avec une prédominance des sciences sociales, cette figure illustre la grande diversité des ancrages disciplinaires (23 disciplines) issus des différents champs scientifiques tels que les sciences humaines et sociales, les sciences naturelles, les sciences médicales, ainsi que les sciences et techniques de l'ingénieur.
Il est intéressant de noter que la question de l'identité disciplinaire n'a pas été a priori problématisée dans cette première phase de recueil de données, dans la mesure où il est apparu relativement facile de repérer une identité disciplinaire principale pour chaque chercheur. Cette apparente évidence dans l'attribution d'une discipline par chercheur, tant de la part des enquêteurs que des enquêtés, a toutefois été remise en question lorsque les chercheurs ont été amenés à y réfléchir de manière plus approfondie, via le questionnaire (avec des questions ouvertes), les entretiens semi-directifs et les focus groups. Les résultats révèlent en effet que l'identité disciplinaire des chercheurs est bien plus complexe et dynamique que ce que laissent paraître, à première vue, les marquages d'appartenance académiques et institutionnels. Cette complexité ressort fortement lorsqu'on se focalise sur les 
Tableau. Nombre de disciplines principales des participants chercheurs (Questionnaire).

\begin{tabular}{|l|l|}
\hline Nombre de disciplines principales & Personnes \\
\hline Une discipline principale & 27 \\
\hline Deux disciplines principales & 17 \\
\hline Trois disciplines principales & 13 \\
\hline Quatre disciplines principales & 7 \\
\hline
\end{tabular}

trajectoires des chercheurs et le processus de construction des identités disciplinaires.

Les chercheurs ont ainsi été invités à indiquer leur(s) discipline(s) principale(s) actuelle(s) (une question ouverte du questionnaire). Cet exercice d'autoréflexion sur leur appartenance disciplinaire révèle que presque la moitié des chercheurs (27 sur $65^{7}$ ) indique une seule discipline principale (Tab.). Cela explique, de manière générale, que l'identité d'un certain nombre de chercheurs pratiquant la recherche interdisciplinaire se construit sur et à partir d'un fondement disciplinaire qui demeure relativement stable. Une autre partie substantielle de chercheurs (17) mentionne une double appartenance disciplinaire, se situant à la fois dans et à l'interface de deux disciplines. Il est enfin intéressant de noter qu'ils sont treize à se réclamer de trois disciplines et sept de quatre disciplines, témoignant par là de l'émergence d'identités multiples. Parmi ces derniers, qui semblent les plus fortement « multidisciplinaires » (4 disciplines principales), la plupart d'entre eux (6 sur 7) appartiennent aux sciences sociales (4) et aux sciences humaines (2). Sans faire de généralisation tant les configurations sont multiples, on peut néanmoins souligner que la tendance au marquage multidisciplinaire de la part des chercheurs relevant des sciences humaines et sociales se confirme pour les bi-, voire les tri-disciplinaires. L'amplitude des variations est particulièrement intéressante lorsque par exemple un chercheur issu des sciences sociales indique quatre disciplines principales (migration studies, sociologie, histoire, sciences politiques) alors qu'un autre chercheur indique une seule appartenance disciplinaire (digital humanities) tout en se réclamant de trois champs scientifiques (sciences humaines, sciences sociales, et sciences et techniques de l'ingénieur).

La question des identités multiples du chercheur impliqué dans la recherche interdisciplinaire soulève également des enjeux méthodologiques de compatibilité plus ou moins grande entre des approches différentes inhérentes aux diverses cultures scientifiques. Le participant chercheur $\mathrm{E}^{8}{ }^{8}$ souligne par exemple la polysémie du terme $\mathrm{d}^{\prime}$ " interdisciplinarité » selon le contexte dans lequel il s'inscrit : "Les études sociales des sciences et de la médecine n'étant pas a priori disciplinaires, il est

7 Un participant-chercheur a indiqué n'avoir aucune discipline principale. normal d'utiliser les outils de la linguistique, sémiotique, histoire, socio, etc. Il en va autrement lorsqu'il s'agit de collaborer avec des quantitativistes issus des recherches sur la santé publique, les médecins, les ingénieurs, etc. » (E3, Entretien). Ces énoncés font allusion aux éventuelles difficultés engendrées dans les collaborations interdisciplinaires entre "sciences molles »- nous dirions plutôt douces - et "sciences dures », entre méthodes qualitatives et quantitatives, entre nature et société. Bien que la mise en œuvre de l'interdisciplinarité soulève des difficultés voire des incompréhensions entre les cultures scientifiques, il ressort au final que l'identité disciplinaire en tant que telle n'est pas mise en crise. L'affirmation d'une identité disciplinaire est en effet souvent renforcée par les processus d'évaluation institutionnelle des carrières des chercheurs qui doivent se démarquer dans un contexte multiculturel et concurrentiel de la production scientifique (Joulian et al., 2005). Cette démarcation pratiquée par la majeure partie des scientifiques répond à un besoin de "sécurité intellectuelle » et de " réussite professionnelle » garantie par $l^{\prime}$ « excellence disciplinaire reconnue » (Kalaora et Larrère, 1989, p. 85). L'identité disciplinaire demeure apparemment le socle à partir duquel se construit le dialogue entre les disciplines, laissant s'exprimer ce que Petrie (1992) avait repéré comme un « paradoxe disciplinaire » selon lequel l'interdisciplinarité serait nécessaire, mais ne pourrait se réaliser sans le concours, également nécessaire, des disciplines.

\section{Rôle des compétences disciplinaires dans l'interdisciplinarité}

Un des débats récurrents en matière d'interdisciplinarité concerne la tension entre la disciplinarité et l'interdisciplinarité (Darbellay et Paulsen, 2011). Cette controverse est non seulement d'ordre théorique, mais aussi $d^{\prime}$ ordre pratique dans les collaborations entre chercheurs. À la question de savoir ce que représente l'interdisciplinarité en regard de leurs compétences disciplinaires (Fig. 2), les participants-chercheurs de notre enquête reconnaissent largement que l'interdisciplinarité est un complément, une plus-value et un moyen de repenser forces et faiblesses de leurs compétences disciplinaires. Globalement, l'interdisciplinarité n'est pas perçue comme un frein au développement des compétences disciplinaires. L'interdisciplinarité comporte ainsi essentiellement deux faces : $c^{\prime}$ est à la fois une forme de critique des cloisonnements et limites disciplinaires, mais aussi de manière plus nuancée une approche qui

\footnotetext{
8 Comme indiqué dans la note 4, l'anonymat est respecté : les locuteurs sont identifiés par une lettre majuscule suivie d'un nombre qui renvoie à leur institution d'appartenance.
} 


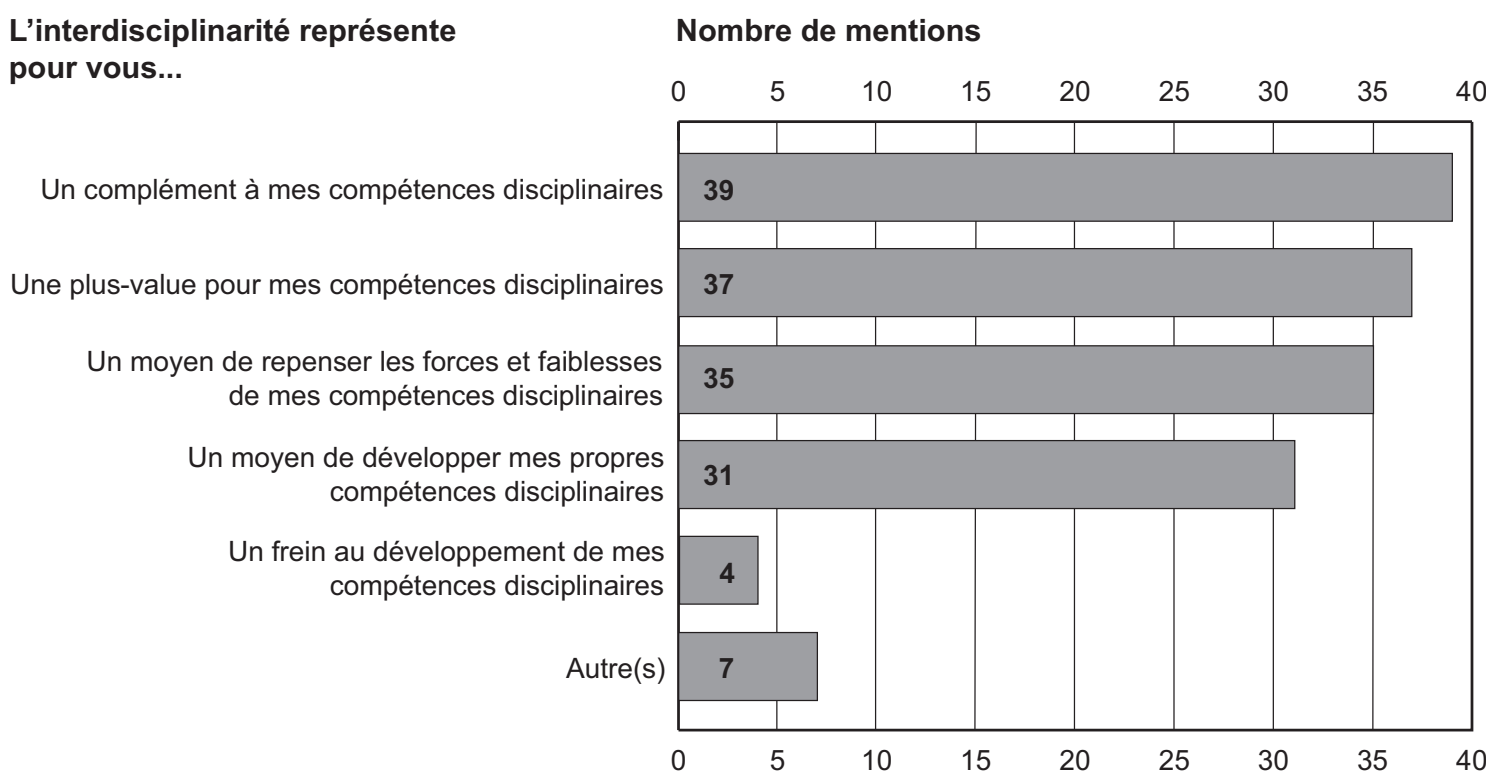

Fig. 2. Compétences disciplinaires et interdisciplinarités.

ne vise pas forcément la remise en cause radicale des disciplines.

Il n'y a pas de contradiction majeure entre la disciplinarité et l'interdisciplinarité dans les dires des chercheurs. Selon A1 par exemple, la perspective interdisciplinaire se développe en prenant appui sur une «base disciplinaire » : «L'interdisciplinarité ne met pas la disciplinarité dans la poubelle. Moi, je dis à mes étudiants qu'on a besoin de la disciplinarité pour faire l'interdisciplinarité ou la multidisciplinarité » (A1, Focus group).

Au-delà de cette vision pacifiée dans les rapports entre disciplinarité et interdisciplinarité, les données plus qualitatives, recueillies dans les commentaires sollicités dans le questionnaire, renforcent cette perception, tout en nuançant et en mettant en évidence les obstacles et difficultés inhérents au travail interdisciplinaire. À ce titre, E3 conçoit par exemple l'interdisciplinarité comme un complément et une plus-value pour le développement de ses compétences disciplinaires et comme un moyen d'en repenser les forces et les faiblesses. Il indique, en revanche, que l'interdisciplinarité peut être un éventuel obstacle sur le plan des carrières académiques, "éventuellement, un frein car souvent les postes/les requêtes, etc. sont disciplinaires » (E3, Questionnaire).

\section{Identités (inter)disciplinaires : stables et provisoires}

L'identité des chercheurs évolue, comme celle de la plupart des acteurs professionnels, en fonction des mutations sociales, économiques, politiques et culturelles dans une communauté de pairs où règne un certain individualisme social (De Singly, 1998 ; Dumont, 1983). Les questions concernant l'identité professionnelle et la manière d'être des " universitaires » (Musselin, 2008) dans une perspective d'échange et de collaboration avec d'autres collègues se sont révélées centrales dans le cadre de notre recherche. Au sens de Dubar (1991), une identité peut être décrite comme une entité présentant une double face : «L'identité n'est autre que le résultat à la fois stable et provisoire, individuel et collectif, subjectif et objectif, biographique et structurel, des divers processus de socialisation qui, conjointement, construisent les individus et définissent les institutions » (Dubar, 1991, p. 113). Cette vision dialectique de l'identité permet de penser des identités paradoxales, à la fois stables et instables, subjectives et objectives, unes et diverses, qui prennent tout leur sens dans le contexte particulier du chercheur disciplinaire qui s'ouvre à l'interdisciplinarité. On peut alors concevoir l'identité (inter)disciplinaire d'un chercheur comme un produit des socialisations successives dans sa trajectoire académique, au cours de laquelle différents environnements (social, institutionnel, humain ou matériel) influencent la construction, la déconstruction et la reconstruction de soi. Il s'agit d'un processus d'aller-retour entre l'identification et la différenciation de soi par rapport à autrui, par rapport au monde : 1'altérité travaille au cœur de l'identité du sujet lui-même (Ricœur, 1990). Les identités de chercheurs pleinement investis dans l'interdisciplinarité sont en fait multiples, mobiles et évolutives : elles se déroulent selon les opportunités de collaborations qui se coconstruisent dans les parcours académiques. A1 indique par exemple 
explicitement dans sa réponse au questionnaire que sa discipline principale est l'écologie humaine. Parallèlement, lors de son entretien approfondi, il partage le récit de son parcours académique et de ses expériences de recherche interdisciplinaire, dessinant un profil hybride et ayant également des compétences multidisciplinaires en architecture et en sciences sociales. Sous l'angle de sa socialisation dans le groupe de recherche, il est intéressant d'entendre ses deux collègues (E1 et D1) dans le cadre de leur propre entretien. Ils témoignent de leurs différentes perceptions de l'identité de A1, vue effectivement comme multiple et tout en se centrant sur la géographie et l'écologie humaine : "Il y a (nom de A1) qui est à la fois je crois sciences politiques, anthropologie et architecture » (E1, Entretien) ; « Professeur (nom de A1) est plutôt géographe, écologie humaine » (D1, Entretien). L'identité du chercheur, en tant qu' « ensemble de significations » (Mucchielli, 1986), est ainsi co-construite dans et par l'interaction avec d'autres chercheurs de sa / ses discipline/s et/ou d'autres disciplines. Son identité est mise en relation dynamique avec d'autres regards/ perceptions au cours de son parcours personnel et professionnel, et elle est amenée à varier en fonction des contextes et des rencontres. Dans le parcours réel d'un chercheur engagé dans l'interdisciplinarité, son identité se reconfigure en fonction du contexte et change sous le regard de l'autre. Cette vision dynamique des identités multiples des chercheurs qui pratiquent l'interdisciplinarité évoque les travaux de Barth (1998) sur l'identité ethnique comme auto-attribution qui se focalise sur les «frontières » ethniques plutôt que sur la constitution interne des groupes ethniques. C'est bien les interactions entre groupes (frontières) qui peuvent définir l'identité ethnique et non seulement la cohérence interne de chacun des groupes.

\section{Profils types : identités disciplinaires et interdisciplinaires}

Forts de ce constat de la multiplicité des identités de chercheurs, nous avons tenté de repérer différents profils types identitaires. Ces profils types ne sont pas des catégories figées, ils doivent au contraire être pensés comme des idéaux types dans l'optique wébérienne. Ils constituent un instrument d'intelligibilité fondamental pour guider notre réflexion (Weber et Freund, 1965). Ces profils types recouvrent des «formes identitaires» (Dubar, 1998) qui vont de la revendication d'appartenance à une seule discipline à des formes plus interdisciplinaires, voire indisciplinées. La construction des profils types de chercheurs a bénéficié, d'une part, de l'analyse de notre corpus des entretiens et focus groups et, $\mathrm{d}$ 'autre part, de nos propres expériences interdisciplinaires en interaction dans le cadre de ce projet. Cette approche compréhensive n'a pas la prétention de couvrir tous les cas de figure qui pourraient se manifester dans le large spectre des expériences de recherche interdisciplinaire. Elle a pour simple objectif de proposer un cadre de réflexion pour mieux comprendre les pratiques. Les profils types se présentent comme suit, sur un continuum allant de la disciplinarité à l'indisciplinarité : l'identité disciplinaire ; les identités interdisciplinaires, déclinées en identité thématique, identité hybride, « interdisciplinary native » et « interdisciplinary migrant »; et enfin l'identité indisciplinée.

\section{Identité disciplinaire}

Les chercheurs qui ont participé aux focus groups de notre recherche se sont présentés lors du tour de table qui inaugure souvent les rencontres universitaires. Outre leur appartenance institutionnelle et/ou leur fonction académique (" membre de...", «professeur ou Dr en...», «directeur de...», etc.), ils ont pour la grande majorité affiché une identité disciplinaire de base : «j'ai une formation de psychologue », "je suis ethnologue», etc. Ils occupent une posture identitaire que nous rapportons au profil type $\mathrm{d}^{\prime}$ «identité disciplinaire», en ce sens qu'ils affirment explicitement un ancrage disciplinaire, tout en pratiquant une recherche ouverte au dialogue avec les autres disciplines: "Je me vois vraiment comme un mathématicien! C'est là que je suis compétent. La sociologie, c'est des trucs que j'ai bricolés » (G3, Entretien). L'affirmation de l'identité disciplinaire ne se fait toutefois jamais par opposition à l'interdisciplinarité, celle-ci est même incluse dans le champ d'investigation dans une perspective relationnelle et dynamique : la philosophie est «complètement interdisciplinaire, par essence » (B4, Entretien) ; elle fait en ce sens partie des «discipline[s] de l'interdisciplinarité » pour reprendre l'expression de Mathieu (1992).

\section{Identité thématique}

L'identité des chercheurs peut aussi s'exprimer, non seulement sous le régime de la disciplinarité, mais sous une forme que nous qualifierions de thématique interdisciplinaire. Les chercheurs ne s'identifient pas à une seule discipline, mais à un champ thématique d'études interdisciplinaires qui est souvent transversal à plusieurs disciplines ou sous-disciplines (par exemple les gender studies, les migration studies, le développement durable, la santé publique, etc.). Ils orientent leurs activités de recherche, $\mathrm{d}$ 'enseignement et de publication selon leurs centres d'intérêt thématiques plutôt que de suivre une trajectoire académique plus classiquement disciplinaire : " J'ai commencé à travailler avec une orientation thématique et notamment quand il s'agit des migrations [...]. Ce n'est pas devenu une discipline, mais c'est devenu un champ énorme où il y a des personnes issues de disciplines très très diverses » ( $\mathrm{A} 5$, Entretien). 


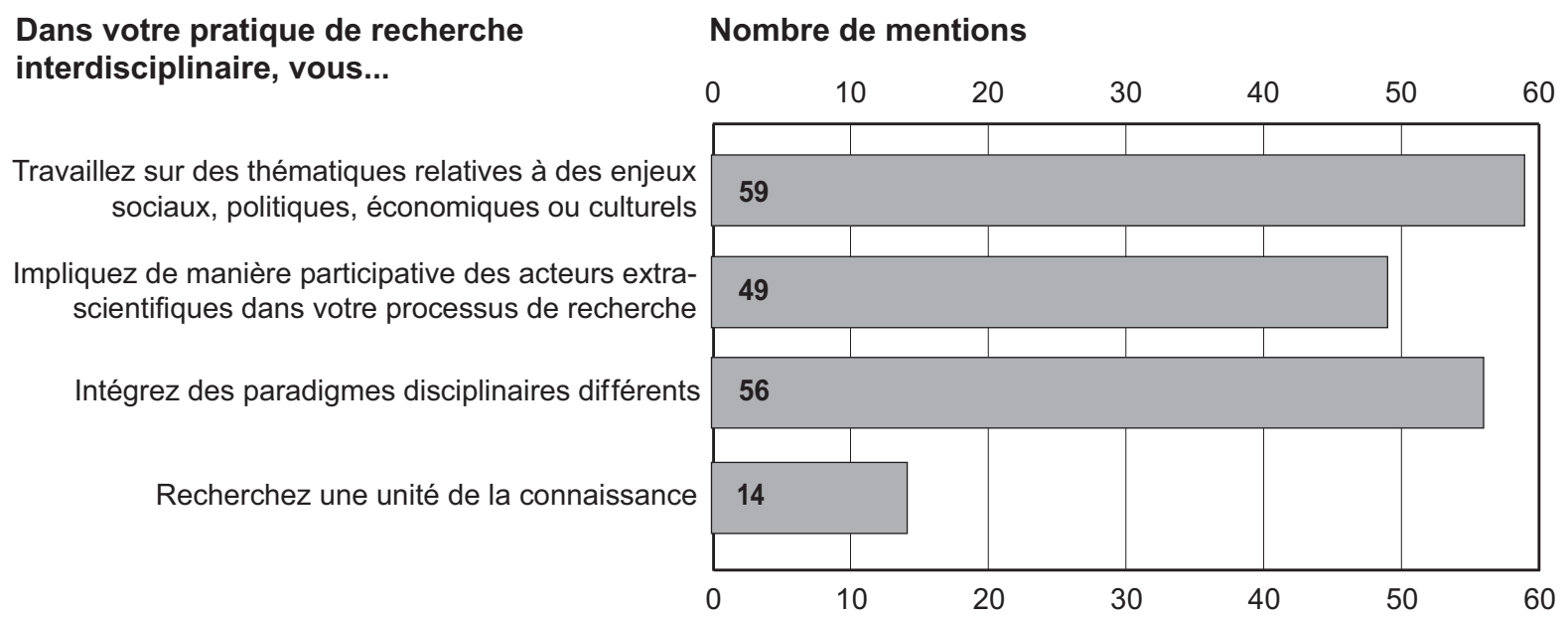

Fig. 3. La recherche interdisciplinaire en pratique.

L'entrée par des thématiques complexes est fréquente dans les recherches interdisciplinaires : il s'agit de porter ses analyses sur des problématiques transversales (sociales, politiques, économiques, naturelles, etc.) qui sont irréductibles à un seul point de vue disciplinaire et qui réclament l'intégration de plusieurs paradigmes scientifiques, et un dialogue entre science et société. Les réponses suivantes apportées au questionnaire (Fig. 3) confirment cette importance accordée aux thématiques transdisciplinaires impliquant des acteurs extrascientifiques. Il ne s'agit pas tant de viser absolument une unification des connaissances disciplinaires (entrer dans un problème par le seul regard des disciplines), mais, au contraire, de mettre l'accent sur des approches pratiques, susceptibles de résoudre des problèmes complexes: "Pour moi, c'est ce qu'on entreprend, ces approches-là et en ayant une thématique donc c'est toujours une approche très pragmatique " (C5, Entretien).

\section{Identité hybride}

Une identité hybride se donne à lire dans les trajectoires de chercheurs qui résultent d'un croisement de deux ou plusieurs disciplines et qui présentent, le plus souvent, un mixte de disciplines antérieures (par exemple la psychosociologie, la socioanthropologie, etc.) et la création d'une nouvelle identité (inter)disciplinaire par hybridation. Dans la construction de cette identité, le chercheur doit maîtriser deux ou plusieurs compétences disciplinaires. C2 a par exemple commencé à faire ses études en biologie et de la recherche en neurosciences, il a ensuite fait une deuxième formation en sociologie et en anthropologie. Il exprime une double identité en biologie et socioanthropologie, et il travaille actuellement sur la sociologie des sciences et des techniques: "Je dirais que mon ancrage principal, quand même, c'est maintenant la sociologie, l'anthropologie. Mais je me considère comme vraiment interdisciplinaire. C'est sûr ! Parce que sur mes terrains, mes deux identités sont absolument indispensables » (C2, Entretien). Certains chercheurs ont bénéficié de doubles ou de multiples formations disciplinaires de base qui les conduisent, par la suite, à développer des recherches interdisciplinaires. Par exemple, le parcours académique de F5 se fonde sur les sciences politiques et la géographie humaine qui sont reliées à une thématique spécifique, à savoir la "planification urbaine ». Celui-ci explique qu'il y a dans certaines circonstances des possibilités d'intégrer différentes disciplines dans un même champ scientifique: "Les barrières disciplinaires $m^{\prime}$ 'ont toujours semblé relativement perméables dans le contexte des sciences sociales et n'ont pas constitué d'obstacle à mon passage de la science politique à la géographie humaine » (F5, Questionnaire).

\section{«Interdisciplinary native »}

Nous proposons de dénommer par l'expression « interdisciplinary native » le chercheur ayant une trajectoire interdisciplinaire dès l'origine de sa formation de base, sans ancrage disciplinaire fixe et dont les intérêts portent sur des domaines scientifiques incluant un large spectre de disciplines différentes. Cette notion de "native » se réfère, par analogie à la notion de «digital natives », à une catégorie générationnelle qui comprend les jeunes nés après 1983 et ayant notamment des compétences poussées en technologie de l'information (Bennett et al. 2008 ; Hargittai, 2010 ; Jones et al., 2010). Comme pour la notion de "digital natives", il faut souligner que les éventuels effets générationnels sont pris en compte dans l'idée d' «interdisciplinary native», ce qui permet d'ailleurs une différenciation par rapport au profil plus large du "interdisciplinary migrant » (voir ci-après). C5, un jeune chercheur ayant une formation initiale en sciences politiques et une thèse de doctorat en sciences humaines et sociales, raconte que l'interdisciplinarité est 
pour lui quelque chose d'évident, «allant de soi » : «Je me suis jamais vraiment posé la question de la disciplinarité, de ce que je faisais, parce que déjà à la base, même je disais en sciences politiques, ça n'a jamais été disciplinaire » (C5, Entretien). Une des caractéristiques de ces chercheurs est qu'ils portent, dès le début de leurs études, un grand intérêt personnel à un large spectre de disciplines différentes. F8, dans la même veine, expose son intérêt initial pour plusieurs disciplines (philosophie, mathématiques, histoire, chimie) et non pas pour une discipline spécifique. Ses intérêts se situent à l'intersection des sciences humaines et des sciences naturelles, ce qui l'a amené par exemple à s'intéresser un temps à la géographie (physique et humaine) qui couvre ces deux cultures scientifiques. Il s'est positionné dès le début de son parcours académique dans l'interdisciplinarité. Il l'a vécue comme une nouvelle culture dans ses travaux individuels et dans la collaboration avec d'autres collègues, en analysant, par exemple, le fonctionnement du système d'organisation du travail collaboratif au sein d'un programme de recherche interdisciplinaire : «Au sein de la géographie j'avais une opportunité... dans le cadre du (nom du programme de recherche) dans lequel j'ai fait mon travail de diplôme, sur la question comment organiser un tel programme avec douze disciplines » (F8, Entretien).

Il n'en demeure pas moins que cette culture interdisciplinaire, particulièrement mise en visibilité chez les interdisciplinary natives, même si elle est fortement promue et encouragée, manque de reconnaissance et de soutien substantiel de la part des institutions universitaires. Certes, cette question de la reconnaissance touche tous les chercheurs en contexte interdisciplinaire, mais plus particulièrement les jeunes chercheurs qui débutent dans l'interdisciplinarité et qui peuvent souffrir d'un «manque de légitimité institutionnelle » (Bühlera et al., 2006). L'interdisciplinarité peut alors devenir elle-même un obstacle et révéler des difficultés institutionnelles, notamment pour la carrière académique. À ce titre, un doctorant en « sciences de la vie » (D2) travaillant sur des questions d'éthique exprime clairement ses incertitudes identitaires en matière de formation et de reconnaissance académiques. Situé dans un contexte institutionnel finalement très disciplinaire, il se questionne : "Moi-même je ne saurais pas vraiment comment me définir. Le problème avec l'éthique c'est que beaucoup de gens disent "éthicien, ça n'existe pas...". En même temps, c'est la seule définition que je pourrais trouver qui me convient, et puis d'un autre côté, le problème c'est évidemment celui de la reconnaissance » (D2, Entretien).

\section{« Interdisciplinary migrant »}

À la différence du interdisciplinary native, le migrant désigne un profil type sans se limiter à un effet d'âge et de génération. Il s'agit le plus souvent de chercheurs confirmés ayant une origine disciplinaire, mais qui s'ouvrent progressivement à l'interdisciplinarité par emprunt et circulation entre les disciplines au cours de leur trajectoire académique. Cette identité se construit en passant par des changements et des transformations $\mathrm{d}^{\prime}$ une discipline à une autre, par migrations successives et nomadisme (Darbellay, 2012 ; Stengers, 1987). A3, ayant une formation initiale d'ingénieur et une thèse en informatique, déclare que les « digital humanities » constituent sa discipline principale actuelle. Il témoigne de son parcours qui a évolué par « interactions » et « hasards de circonstances » :

« À un moment, j'ai senti qu'il y avait un autre domaine où on pouvait faire ça, c'était dans la psychologie du développement et donc j'ai basculé je dirais... ça a commencé à travailler beaucoup en robotique et en interaction très forte avec des psychologues du développement. Donc, c'était toujours dans l'interdisciplinaire, mais sur un autre domaine. Et c'est la même chose ensuite qui s'est développée à plusieurs reprises et jusqu'à très récemment dans les interactions que j'ai pu voir avec les sciences humaines et sociales et l'histoire. Et c'est ça qui conduit, par les hasards de circonstances finalement, aujourd'hui à mon poste actuel » (A3, Entretien).

Certains chercheurs mentionnent également leur participation à la création de communautés interdisciplinaires avec des collègues de différentes disciplines: «On a inventé notre interdisciplinarité, parce que les médecins s'intéressaient, mais ils ne savaient pas comment faire, les théologiens avaient une expertise en éthique, et puis les philosophes, donc on a commencé à constituer des groupes » (A4, Entretien).

\section{Identité indisciplinée}

L'indisciplinarité consiste à enquêter en dehors des disciplines et à éviter toute sclérose disciplinaire (Catellin et Loty, 2013 ; Legay, 1986 ; Loty, 2005). Ce terme, comme celui de l'interdisciplinarité, ne s'oppose pas par essence à celui de la disciplinarité, mais il défie sans détour les clôtures disciplinaires pour s'en affranchir et se libérer des mises en disciplines de la connaissance. C3, par exemple, ne se considère pas comme « disciplinaire » et indique que sa thèse de doctorat présentait un caractère "indisciplinaire ». Il adopte, en effet, une démarche indisciplinée pour aborder sa problématique de recherche : « $C^{\prime}$ était une thèse assez j'ai envie de dire indisciplinaire, puisqu'elle empruntait à la fois à l'histoire des sciences mais aussi à l'anthropologie, etc. » (C3, Entretien). Il ne se considère pas comme un « homo academicus» et il se positionne en dehors de toute « revendication disciplinaire ». A8 non plus ne s'identifie pas à une discipline et il dit ne pas avoir et ne jamais avoir eu une identité disciplinaire académique, en raison de l'importance accordée à son parcours extraacadémique : il débute en effet son parcours professionnel hors académie, en tant que laborantin en chimie avec 
des travaux interdisciplinaires dans les domaines de la protection des eaux; il reprend ensuite des études en sciences forestières et obtient enfin un poste de directeur $\mathrm{d}^{\prime}$ une institution universitaire. C'est une motivation personnelle qui l'a poussé à s'engager dans un travail interdisciplinaire, dès le début de son parcours professionnel, et qui est liée à son intérêt porté aux problèmes sociétaux dans les domaines de l'environnement. Il a été fasciné par les approches systémiques et la méthodologie: « Déjà à cette époque-là [...], j'ai discerné l'approche systémique là-dedans et j'ai aussi réfléchi sur la méthodologie [...] mais il [le travail interdisciplinaire] est et reste dès le départ un engagement social » (A8, Entretien).

\section{Conclusion}

Les différents profils types identifiés dans cette contribution s'incarnent dans des trajectoires de chercheurs qui vivent au quotidien le paradoxe identitaire qui consiste à être soi-même (se positionner) dans sa discipline, voire dans son indiscipline, tout en étant ouvert à l'autre disciplinaire en créant des interactions et en sortant de sa zone de confort pour faire avancer sa recherche. Comme le dit Legay (1986), il faut en effet protéger à tout prix les zones d'intersection formées, en formation et celles à venir entre les disciplines :

«Ce qui est essentiel, c'est qu'un chercheur découvre la complexité de l'objet de sa recherche, c'est qu'un spécialiste d'une discipline refuse le ghetto de sa discipline en même temps que la position hiérarchique qui lui a été attribuée par rapport aux autres disciplines. En débordant par nécessité des limites prescrites à son domaine traditionnel, le chercheur se donne l'occasion de voir autrement et de rencontrer d'autres collègues qui ont fait la même démarche que lui à partir de leurs propres disciplines. Des zones d'intersection se forment, dont on ne sait pas au départ quelles seront leur importance, leur durée et leur fécondité, mais qu'il faut protéger à tout prix » (p. 395).

Les différents profils types identifiés dans cette contribution participent chacun à leur manière à cette protection du travail interdisciplinaire nécessaire à la production de connaissances nouvelles. Les témoignages des chercheurs que nous avons recueillis démontrent la pluralité des identités disciplinaires dans les pratiques de recherche interdisciplinaire, conçues comme des processus dynamiques, individuels et collectifs. Les identités (inter)disciplinaires des chercheurs se définissent au croisement de divers paramètres: de leur formation de base (disciplinaire, bidisciplinaire, multidisciplinaire ou interdisciplinaire), en passant par leurs compétences théoriques et méthodologiques, les spécificités de leur trajectoire académique et professionnelle ou encore leurs intérêts personnels. Ce sont autant de variables internes et externes qui façonnent l'être et le devenir identitaire de chacun.
L'interdisciplinarité n'est pas seulement une affaire de compétences cognitives ou pratiques des chercheurs, elle a aussi à voir avec leur plus ou moins forte appartenance à des cultures scientifiques disciplinaires. Comme le montre Henkel (2002), l'identité des chercheurs reste parfois centrée sur leur propre discipline et relativement stable au cours de la période de formation, alors même que les perspectives professionnelles à long terme sont de plus en plus diversifiées et incertaines. Cette diversification est de plus en plus importante, non seulement au niveau de la vie des sciences, mais aussi au niveau individuel des carrières académiques (Ziman, 1987). La question de l'identité disciplinaire renvoie finalement à la diversité, à la complexité et à la mobilité accrue dans les trajectoires des chercheurs qui peuvent appartenir à un ou plusieurs profils types au cours de leur carrière. Bien que les identités gagnent en mobilité et que le contexte culturel et social ambiant de la communication et de la mondialisation amplifie ce processus, on est néanmoins en droit de se poser la question de la place qui est ou sera réellement réservée aux mutants interdisciplinaires à l'université, à moins que ceux-ci soient capables de la transformer de l'intérieur afin que l'interdisciplinarité puisse s'y déployer au même titre que les disciplines.

\section{Remerciements}

Nos remerciements vont au Fonds national suisse de la recherche scientifique (FNS) et à ses responsables qui ont, via le Comité spécialisé en recherche interdisciplinaire (CoSP-ID), soutenu le projet d'étude des pratiques de recherche interdisciplinaire dont nous présentons ici quelques résultats. Nous tenons également à remercier l'ensemble des chercheurs qui se sont prêtés avec confiance au jeu de l'enquête, en nous faisant part de leurs expériences de recherche.

\section{Références}

Barth, F., 1998. Ethnic groups and boundaries: The social organization of culture difference, Long Grove (IL), Waveland Press.

Becher, T., Trowler, P., 2001. Academic tribes and territories: Intellectual enquiry and the culture of disciplines, Milton Keynes (UK), Society for Research into Higher Education \& Open University Press.

Bennett, S., Maton, K., Kervin, L., 2008. The 'digital natives' debate: A critical review of the evidence, British Journal of Educational Technology, 39, 5, 775-786.

Brun, E., Betsch, J.-M., Blandin, P., Humbert, G., Lefeuvre, J.C., Marinval, M.-C., 2007. Postures des scientifiques et interdisciplinarité dans le champ de l'environnement, Natures Sciences Sociétés, 15, 2, 177-185. 
Bühlera, È.A., Cavaillé, F., Gambino, M., 2006. Le jeune chercheur et l'interdisciplinarité en sciences sociales, Natures Sciences Sociétés, 14, 4, 392-398.

Campbell, D.T., 1969. Ethnocentrism of disciplines and the fish-scale model of omniscience, in Sherif, M., Sherif, C.W. (Eds), Interdisciplinary Relationships in the Social Sciences, Chicago, Aldine Transaction, 328-348.

Catellin, S., Loty, L., 2013. Sérendipité et indisciplinarité, Hermès, 67, 32-40.

Charbonneau, S., 2005. Réflexion d'un juriste sur l'enfermement disciplinaire, Natures Sciences Sociétés, 13, 1, 58-61.

Clark, B.R., 1983. The Higher Education System: A Cross-National Perspective, Berkeley (CA), University of California Press.

Creswell, J.W., Plano Clark, V.L., 2007. Designing and Conducting Mixed Methods Research, Thousand Oaks (CA), Sage Publications.

Darbellay, F. (Ed.), 2012. La circulation des savoirs: interdisciplinarité, concepts nomades, analogies, métaphores, Berne, Peter Lang.

Darbellay, F., Paulsen, T. (Eds), 2011. Au miroir des disciplines : Réflexions sur les pratiques d'enseignement et de recherche interet transdisciplinaires, Im Spiegel der Disziplinen. Gedanken über inter- und transdisziplinäre Forschungs und Lehrpraktiken, Berne, Peter Lang.

De Singly, F., 1998. Individualisme et lien social, Lien Social et Politiques-RIAC, 39, 33-46.

Dubar, C., 1991. La Socialisation: construction des identités sociales et professionnelles, Paris, Armand Collin.

Dubar, C., 1998. Trajectoires sociales et formes identitaires : clarifications conceptuelles et méthodologiques, Sociétés Contemporaines, 29, 1, 73-85.

Dumont, L., 1983. Essais sur l'individualisme: une perspective anthropologique sur l'idéologie moderne, Paris, Le Seuil.

Gullestad, M., Lien, M.E., Melhuus, M., 2009. Anthropologie "chez soi » et anthropologie «chez l'autre», Ethnologie Française, 39, 2, 206-215.

Hargittai, E., 2010. Digital na(t)ives? Variation in Internet skills and uses among members of the 'net generation', Sociological Inquiry, 80, 1, 92-113.

Henkel, M., 2002. L'identité des universitaires: quelle évolution ?, Politiques et Gestion de l'Enseignement Supérieur, 14, 3, 159-170.

Jones, C., Ramanau, R., Cross, S., Healing, G., 2010. Net generation or digital natives: Is there a distinct new generation entering university?, Computers $\mathcal{E}$ Education, 54, 3, 722-732.

Joulian, F., de Cheveigné, S., Le Marec, J., 2005. Dossier Interdisciplinarité "Évaluer les pratiques interdisciplinaires", Natures Sciences Sociétés, 13, 3, 284-290.

Kalaora, B, Larrère, G.-R., 1989. Les sciences sociales et les sciences de la nature au péril de leur rencontre, in Mathieu,
N. et Jollivet, M. (Eds), Du rural à l'environnement, la question de la nature aujourd'hui, Paris, ARF/L'Harmattan, 81-89.

Legay, J.-M., 1986. Quelques réflexions à propos d'écologie : défense de l'indisciplinarité, Acta Oecologica, Oecologia Generalis, 7, 4, 391-398.

Lemay, V., Darbellay, F. (Eds), 2014. L'Interdisciplinarité racontée: chercher hors frontières, vivre l'interculturalité, Berne, Peter Lang.

Loty, L., 2005. Pour l'indisciplinarité, Voltaire Foundation, 245259.

Mathieu, N., 1992. Géographie et interdisciplinarité : rapport naturel ou rapport interdit, in Jollivet, M., Sciences de la nature, sciences de la société : les passeurs de frontières, Paris, CNRS Éditions, 129-154.

Mathieu, N., Schmid, A.-F. (Eds), 2014. Modélisation et interdisciplinarité: six disciplines en quête d'épistémologie, Paris, Éditions Quæ.

Morin, E., 1990. Sur l'interdisciplinarité, in Carrefour des sciences, Actes du Colloque du CNRS, Paris, Éditions du CNRS, 21-29.

Mougenot, C., 2011. Raconter le paysage de la recherche, Paris, Éditions Quae.

Mucchielli, A., 1986. L'Identité, Paris, Presses universitaires de France.

Musselin, C., 2008. Les Universitaires, Paris, La Découverte.

Origgi, G., Darbellay, F., 2010. Repenser l'interdisciplinarité, Genève, Éditions Slatkine.

Ouattara, F., 2004. Une étrange familiarité : les exigences de l'anthropologie «chez soi ", Cahiers d'Études Africaines, 3, 635-658.

Petrie, H.G., 1992. Interdisciplinary education: Are we faced with insurmountable opportunities?, American Educational Research Association, 18, 299-333.

Ricœur, P., 1990. Soi-même comme un autre, Paris, Le Seuil.

Stake, R.E., 1995. The Art of Case Study Research, London, Sage Publications.

Steffen, G., Sedooka, A., Paulsen, T., Darbellay, F., 2015. Pratiques langagières et plurilinguisme dans la recherche interdisciplinaire: d'une perspective mono à une perspective pluri, Questions de Communication, 27.

Stengers, I. (Ed.), 1987. D'une science à l'autre: des concepts nomades, Paris, Le Seuil.

Tashakkori, A., Teddlie, C., 2003. Handbook of mixed methods in social $\mathcal{E}$ behavioral research, Thousand Oaks (CA), Sage Publications.

Wainwright, E., Barker, J., Ansell, N., Buckingham, S., Hemming, P., Smith, F., 2014. Geographers out of place: Institutions, (inter)disciplinarity and identity, Area, 410-417.

Weber, M., Freund, J., 1965. Essais sur la théorie de la science, Paris, Plon.

Ziman, J.M., 1987. Knowing Everything about Nothing: Specialization and Change in Scientific Careers, Cambridge, Cambridge University Press. 